\title{
Effect on B Cell Function by Mesenchymal Stem Cells of Different Derivation
}

\section{Erin L Collins*, Maosong Qi and Gary Gilkeson}

Division of Rheumatology and Immunology, Department of Medicine, Medical University of South Carolina, Charleston, SC 29425, USA

\begin{abstract}
Mesenchymal stem cells (MSCs) are multipotent progenitor cells that have broad immunomodulatory properties. The regulatory mechanism of MSCs is of particular relevance due to interests in cell therapy. Although many studies regarding MSC mechanism of action have been reported, MSC effects on B cell proliferation and function is controversial. B cells play an important role in the disease pathogenesis of systemic lupus erythematosus (SLE). If MSCs are to be developed as a cell therapy for lupus it is important to understand the interaction of MSCs and B cells. In this study we revisit and expand studies of MSCs from various sources on healthy and lupus patient CD19+ B cell proliferation and begin to examine the mechanism by which these cells are interacting. Our results clearly indicate that MSCs are able to have significant effects on B cell proliferation and TNFa production. Moreover, MSC do not enhance $\mathrm{B}$ cell proliferation or function in vitro.
\end{abstract}

Keywords: Mesenchymal stem cells; Autoimmunity; Systemic lupus erythematosus; B cells; Cell therapy

Abbreviations: MSC: Mesenchymal Stem Cells; SLE: Systemic Lupus Erythematosus; UC: Umbilical Cord; HBM: Healthy Bone Marrow; LBM: Lupus Bone Marrow

\section{Introduction}

Mesenchymal stem cells (MSCs) are multipotent progenitor cells that can be isolated from various tissues and have broad immunomodulatory properties [1]. The regulatory properties of these cells suggest they are a potential source for cell therapy of numerous diseases. Well studied is the ability of MSCs to impact the function of several immune cells including $\mathrm{T}$ cells and antigen presenting cells [2-4]. However, MSC effects on B cell proliferation and function has been controversial. To date, reports regarding MSCs modulation of B cell function have varied significantly in their outcomes. Bone marrow derived MSCs were found to inhibit B cell proliferation, differentiation, and antibody production [5-8]. Likewise, umbilical cord derived MSCs were able to inhibit B cell proliferation and differentiation [9]. However, Traggiai et al., conversely reported bone marrow MSCs promoted the proliferation and polyclonal expansion of B cells [10].

As B cells play an important role in the disease pathogenesis of systemic lupus erythematosus (SLE), it is essential to understand the interaction of MSCs and B cells if MSCs are to be developed as a cell therapy for lupus. Our recent studies in MRL/lpr lupus prone mice demonstrated variable efficacy of MSCs in modulating disease activity, depending on the source of origin [11]. Moreover, we found that B cells from lupus individuals were unable to inhibit B cell proliferation. In this study we revisit and expand studies of MSCs from various sources on healthy and lupus patient CD19+ B cell proliferation to begin to clarify how these cells are interacting.

\section{Materials and Methods}

\section{Isolation and culture of human MSC}

The use of human UC-MSC and BM-MSC was approved by the IRB of the Medical University of South Carolina (MUSC) and all donors gave informed consent. The MSC were isolated and cultured as previously described with slight modification $[12,13]$. Briefly, human UC-MSC harvested from the Wharton's Jelly in umbilical cords of healthy babies born by $\mathrm{C}$ section at the MUSC hospital were expanded in Alpha-MEM (Gibco) supplemented with $10 \%$ human platelet lysate (Emory University), $2 \mathrm{mM}$ L-glutamine (Lonza BioWhittaker), and antibiotics $(100 \mathrm{U} / \mathrm{ml}$ penicillin and $100 \mu \mathrm{g} / \mathrm{ml}$ streptomycin; Lonza BioWhittaker), and placed in a humidified cell culture incubator containing $5 \% \mathrm{CO}_{2}$ at $37^{\circ} \mathrm{C}$. Non-adherent cells were removed by changing medium every 3 days after the initial plating. After 7 days of incubation, when UC-MSC colonies took up at least $60-80 \%$ of the total surface area, the cells were sub-cultured.

Both healthy and lupus patient bone marrow mononuclear cells were collected from bone marrow aspirates by gradient centrifugation and seeded at a density of $1 \times 10^{6}$ cells $/ \mathrm{cm}^{2}$ in the same media used for UC-MSC. After 3 days of culture, non-adherent cells were removed and the medium was changed twice weekly thereafter. Once $60-80 \%$ confluence was reached, adherent cells were re-plated at a density of $10^{4}$ cells $/ \mathrm{cm}^{2}$ in medium for expansion. Human BM-MSCs were derived from fresh whole bone marrow of healthy donors (ALLCELLS) and 4 lupus patients. Lupus patients consisted of 3 female and 1 male African Americans between the ages of 28 to 41 . Two patients had active disease with Systemic Lupus Erythematosus Disease Activity Index (SLEDAI) scores $>6$. 2 patients had inactive disease with a SLEDAI score $<2$. All patients were on prednisone with a dose $\leq 5 \mathrm{mg}$ and either on azathioprine or mycophenylate.

After three passages, the cells were harvested. MSC were identified according to the criteria of the International Society for Cellular Therapy [14]. Briefly, flow cytometric analysis confirmed the cells expressed CD44, CD90, and CD105, but not CD31, CD45, or HLA-DR.

*Corresponding author: Erin L Collins, Division of Rheumatology and Immunology, Department of Medicine, Medical University of South Carolina, Charleston, SC 29425*, USA, Tel: 843789-6798; Fax: 843876-5131; E-mail: collinel@musc.edu

Received March 04, 2015; Accepted July 23, 2015; Published July 25, 2015

Citation: Collins EL, Qi M, Gilkeson G (2015) Effect on B Cell Function by Mesenchymal Stem Cells of Different Derivation. J Stem Cell Res Ther 5: 294 doi:10.4172/2157-7633.1000294

Copyright: $\odot 2015$ Collins EL, et al This is an open-access article distributed under the terms of the Creative Commons Attribution License, which permits unrestricted use, distribution, and reproduction in any medium, provided the original author and source are credited. 
The capacity of MSC to differentiate along adipogenic and osteogenic lineages was evaluated as described previously [15]. Cells of passage 3-6 were used in the experiments. Initial MSC confirmation also confirmed purity of the cell lines. MSC purity in cultures post cryo preservation, prior to use, was determined by additional flow confirmation of MSC specific markers and absence of non-MSC markers. In all experiments, analyses indicated $>95 \%$ purity.

\section{B Cell proliferation assay}

Peripheral blood mononuclear cells from the blood of healthy donors $(n=5)$ were isolated using lymphocyte separation medium (Cellgro). CD19+ B cells were isolated by CD19 microbeads (Miltenyi) according to the manufacturer's instructions. B cells were labeled with $2 \mu \mathrm{M}$ carboxyfluorescein diacetate succinimidyl ester (CFSE) (Invitrogen). $5 \times 10^{4} \mathrm{CD} 19+$ cells were co cultured with $5 \times 10^{4} \mathrm{MSCs}$ from various sources in a 96-well flat bottom plate (Costar) with a total volume of 200ul RPMI 1640 (Cellgro) supplemented with $10 \%$ fetal bovine serum, $2 \mathrm{mM} \mathrm{L}$-glutamine, $55 \mu \mathrm{M} 2$-mercaptoethanol (Gibco), $100 \mathrm{U} / \mathrm{ml}$ penicillin and $100 \mu \mathrm{g} / \mathrm{ml}$ streptomycin. B cells received the following stimuli: $2.5 \mu \mathrm{g} / \mathrm{ml} \mathrm{CpG}$ oligonucleotide 2006 (Miltenyi), $1 \mu \mathrm{g} /$ $\mathrm{ml}$ soluble CD154 (CD40L) (BioLegend), $2.5 \mu \mathrm{g} / \mathrm{ml} \mathrm{F(ab')2} \mathrm{anti-human}$ $\mathrm{IgM} / \mathrm{IgA} / \mathrm{IgG}$ (Jackson ImmunoResearch) and 1000U/ml interleukin 2 (IL2) (BioLegend). 3 UC-MSC lines, 2 HBM-MSC lines, and 3 LBMMSC lines were tested 1-2 times. Plates were incubated for 4 days at $37^{\circ} \mathrm{C}$ in a humidified atmosphere with $5 \% \mathrm{CO}_{2}$. CD19+ cells were then collected, stained with anti-human PE-labeled CD19 (Miltenyi). Cell proliferation status was analyzed by flow cytometry.

\section{Cytokine analysis}

Human IL6 and TNFa ELISAs were performed on supernatants from proliferation assays. ELISA kits were obtained from BioLegend and the procedure was followed to the kit's specifications. Plates were read using a Multiskan Ascent at $450 \mathrm{~nm}$.

\section{Statistical analysis}

All data were analyzed using Prism version 5.0 software (GraphPad, San Diego, CA). One-way ANOVA (Tukey posttest) was utilized to test for significance between groups for in vitro assays. A 95\% confidence limit, defined by $p$ values $\leq 0.05$, was considered to be statistically significant and indicated within the figures.

\section{Results and Discussion}

Human MSCs from healthy donors are effective in ameliorating disease in lupus prone mice [11,16-20]. However, there is limited understanding of the mechanism by which these cells elicit their effect. Hyperactivity and autoantibody production by B cells is a trademark for immunological disorders such as SLE [21]. With conflicting data surrounding the ability of MSCs to modulate B cell functions these experiments aim to assess the ability of MSCs, from various sources, to inhibit B cell proliferation.

An earlier study showed that bone marrow derived MSCs increased the proliferation of CD19+ B cells [10]. We repeated these studies with MSCs derived from bone marrow and umbilical cords to examine if these effects were dependent on the MSC source of origin. Our data demonstrates that healthy human MSC from bone marrow and umbilical cords suppressed healthy B cell proliferation to a greater capacity than lupus bone marrow MSCs, albeit not reaching significance (Figure 1A). As in a previous study [22], the lupus donor MSCs trended towards reduced capacity to suppress healthy B cell proliferation when compared to healthy donor MSC (Figure 1A).
These data support the suppressive, not the proliferative, findings of previous studies.

B cells play an important role in the in the pathogenesis of SLE thus we examined whether MSCs from the different sources were able to impact B cell proliferation from lupus donors. When examining CD19+ B cells from lupus patients we found a blunted B cell proliferative response to immune stimuli, which is a limitation of this study (Figure 1B). This restricted proliferation is likely due to patients being on immunosuppressive drugs at the time of $B$ cell isolation or that, like T cell populations, the lupus B cells are "exhausted" and unable to proliferate. Traggiai et al. stated that bone marrow derived MSCs from healthy donors were unable to inhibit the B cell proliferation of SLE patients [10]. Similarly, we also found that healthy bone marrow derived MSCs did not inhibit lupus B cell proliferation. Unlike past studies, we did not see a significant increase in B cell proliferation [10]. Moreover, no effect on lupus B cell proliferation was observed in co-cultures of MSCs from lupus patients or healthy controls. However, MSCs derived from umbilical cords did reduce lupus B cell proliferation, although, not achieving significance (Figure 1B).

TNFa is a cytokine that is made by B cells and can promote their proliferation. Additionally, TNFa is an activating cytokine for MSCs [23]. Upon stimulation with $\mathrm{TNF} \alpha, \mathrm{MSCs}$ increase production of suppressive cytokines, such as IL6, NO, IDO, and PGE2 [24]. A key cytokine produced by activated MSCs, IL6, can inhibit TNFa. We measured TNFa and IL6 in the supernatants of our cultures to determine if these cytokines could explain the differences in suppressive function by MSCs from different sources. Umbilical cord and healthy bone marrow derived MSCs made significantly more IL6 when cocultured with healthy donor B cells then B cells cultured alone (Figure 2A). Lupus bone marrow MSCs also produced more IL6 in co-culture in comparison to $\mathrm{B}$ cells alone, although it was not significant (Figure 2A). In culture with lupus B cells, all sources of MSCs produced IL6, though the amount was significantly less than when these cells were cultured with healthy B cells (Figure 2A). There was no difference in IL6 production between the different MSC groups when cultured with lupus B cells.

MSCs produce IL6 in response to TNFa, thus we hypothesized that the reduced amount of IL6 production by all MSCs co-cultured with lupus B cells was due to a reduced capacity of these lupus B cells to produce TNFa. We did find markedly lower production of TNFa by lupus B cells when compared to healthy B cells (Figure 2B). MSCs from all sources were able to significantly inhibit TNFa production by healthy donor B cells (Figure $2 \mathrm{~B}$ ). Although all MSC subsets did decrease TNFa production by lupus B cells in co-culture, the levels of TNFa produced by the lupus B cells was reduced compared to healthy $\mathrm{B}$ cells (Figure $2 \mathrm{~B}$ ).

MSCs from all sources were able to reduce proliferation and TNFa production in healthy B cells. However, only umbilical cord derived MSCs were able to reduce both proliferation and TNF $\alpha$ production by lupus B cells. Though due to the poor responses of the lupus B cells overall, most of the results comparing B cells alone to B cells plus MSCs were trends and not significant differences. The decreased TNFa production and proliferation seen in lupus B cells, as stated previously, we attribute to either the $\mathrm{B}$ cells being less responsive due to the medications they are receiving or due to the B cells being "exhausted". Diminished TNFa production by lupus B cells may explain the reduced IL6 production by MSCs and subsequent decreased activation in the lupus B cell co-cultures. 
A.

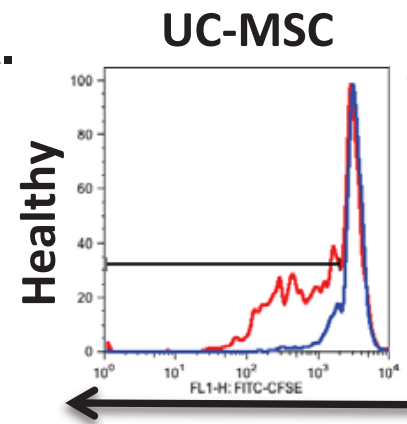

HBM-MSC

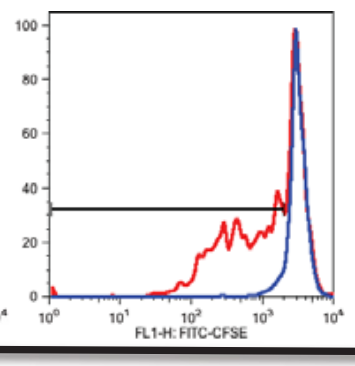

CFSE
LBM-MSC

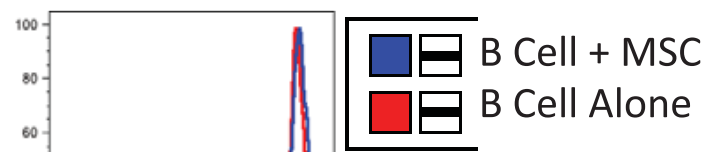

Healthy B Cell Proliferation

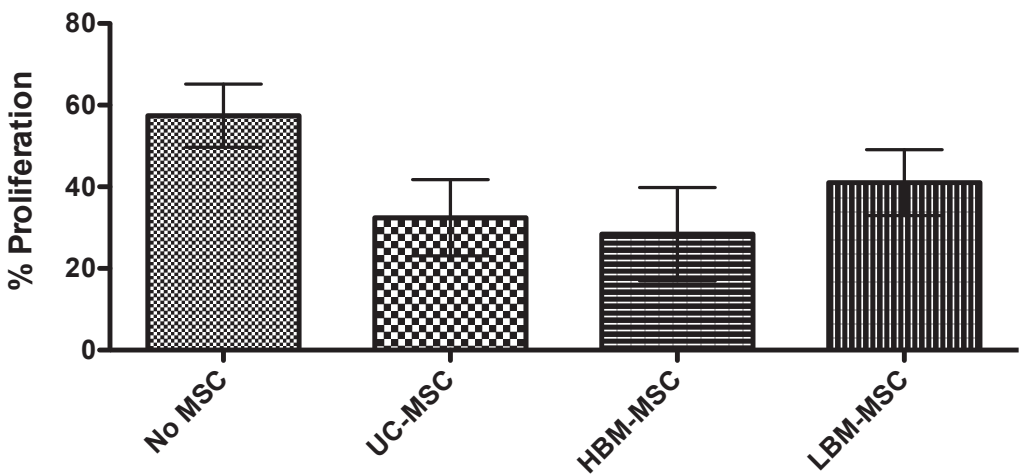

B.

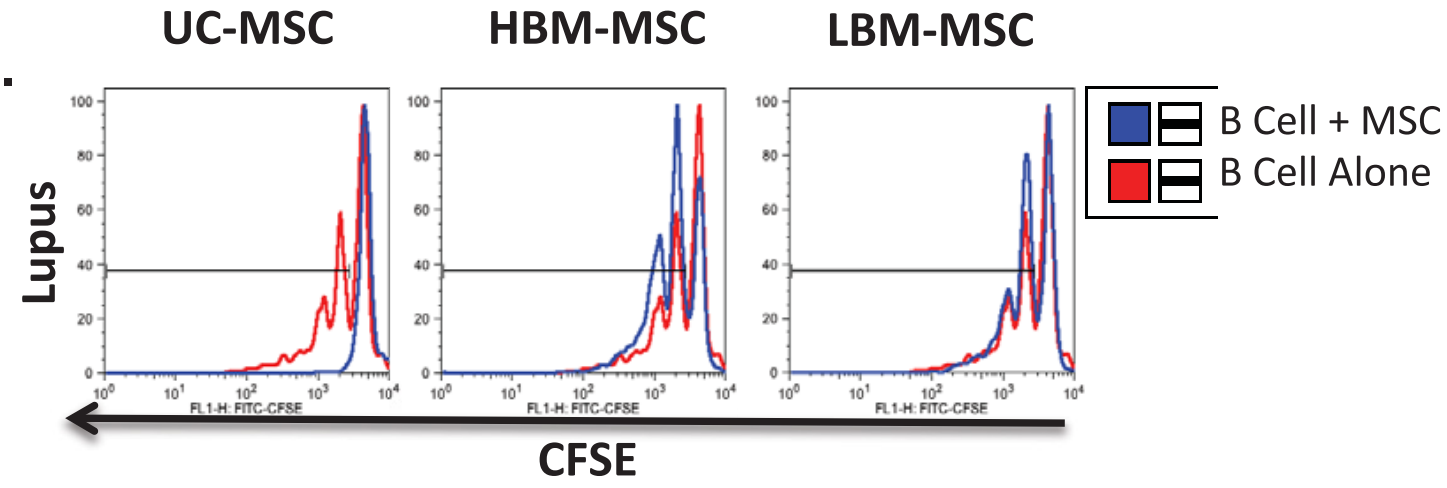

Lupus B Cell Proliferation

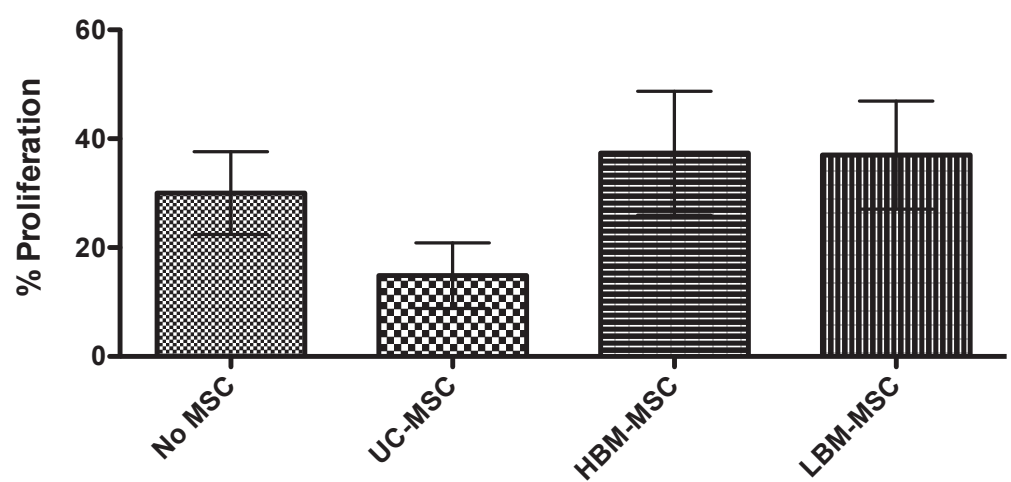

Figure 1: Umbilical cord derived MSC suppress both healthy and lupus B cell proliferation. Healthy and lupus donor CD19+ B cells were collected from MSC coculture assay after 96 hours of incubation. B cells were stained with B cell specific antibodies and analyzed by flow cytometry. Proliferation of CD19+ B cells was measured by CFSE dilution. Data are mean \pm SEM. Statistical comparisons were performed using two-way ANOVA with Bonferroni posttest. * $p \leq 0.05$; $n=5$. Experiments were done with 5 healthy and 5 lupus donors and 2-3 different MSC lines. 
Citation: Collins EL, Qi M, Gilkeson G (2015) Effect on B Cell Function by Mesenchymal Stem Cells of Different Derivation. J Stem Cell Res Ther 5: 294. doi:10.4172/2157-7633.1000294

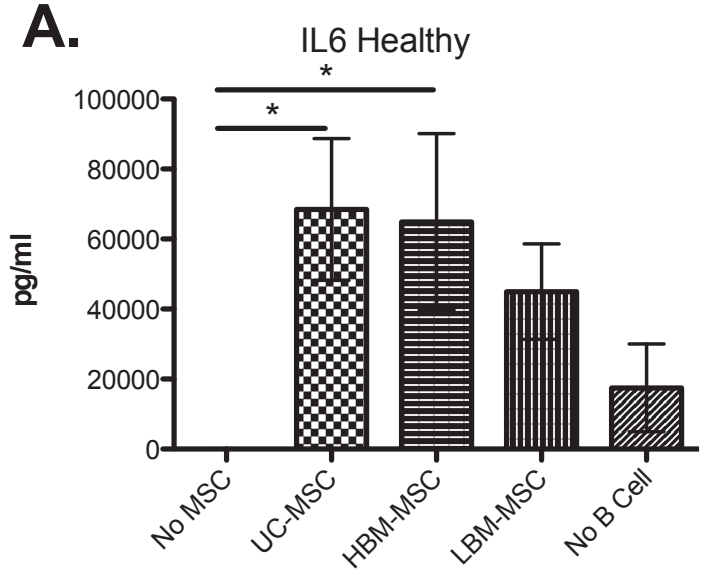

B.

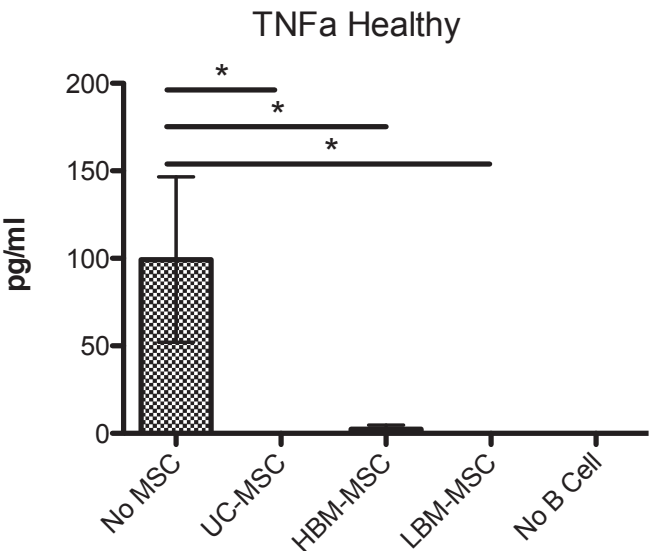

IL6 Lupus

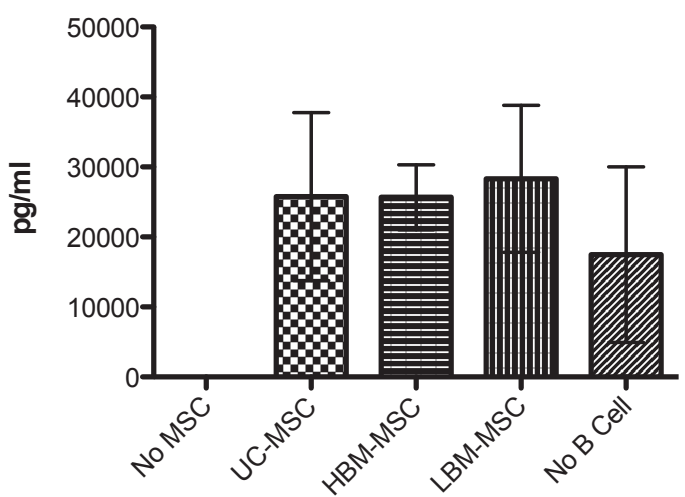

TNFa Lupus

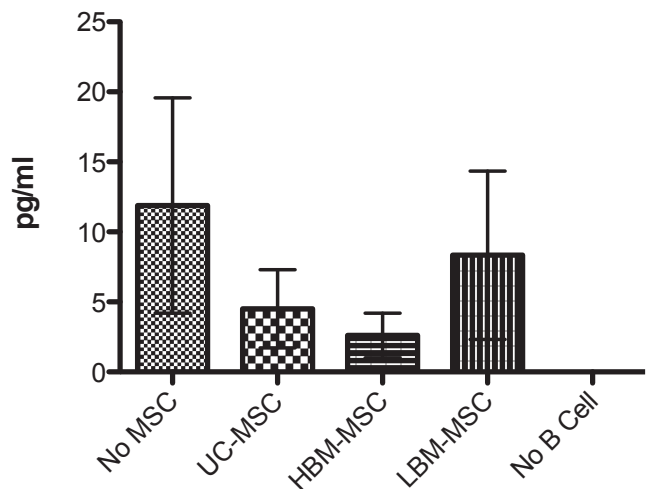

Figure 2: MSC reduce TNFa production by healthy CD19+ B cells. Healthy and Lupus donor CD19+ B cells were co-cultured with MSC from various sources for 96 hours. After 96 hours supernatants were collected. dsDNA autoantibodies and cytokine production was detected via ELISA. Statistical comparisons were performed using one-way ANOVA with Tukey posttest. * $p \leq 0.05 ; n=5$. Experiments were done with 5 healthy and 5 lupus donors and 2-3 different MSC lines.

\section{Conclusion}

In this report, we found that MSC had significant effects on healthy donor B cell proliferation and TNFa production with a trend towards the lupus MSCs having a more modest effect. Lupus B cells had a blunted response to immune stimuli making it difficult to determine significant differences between the different MSC subsets and being a limitation to this study. Regardless, we did not see enhanced proliferation or cytokine production by lupus B cells when co-cultured with MSCs. It is likely that variances in experimental methods explain the differences in results reported to date on the effect of MSC co-culture on B cell proliferation, antibody production, and cytokine production. Recently, a study by Rosando et al. showed that $\mathrm{T}$ cells facilitated $\mathrm{B}$ cell suppression in coculture with MSCs. They showed a lack of B cell suppression by bone marrow derived MSCs, however, when T cells were added suppressive functions were observed that were contact dependent [25]. This paper further demonstrates the variance of results that are obtained depending on culture conditions. They do, however, corroborate our findings that MSCs do not enhance B cell proliferation.

The objective of our study was to improve our understanding of the role of MSCs on B cell function. If MSCs do enhance B cell function, this would be predicted to be deleterious as a therapy for lupus patients. Our results, as well as those of Rosando, clearly indicate that MSCs do not enhance B cell proliferation in vitro. Moreover, in vivo studies of MSC infusions in humans and mice report either no effect on B cell numbers and autoantibody levels or a decrease after MSC treatment. Although more studies need to be performed to decipher the exact mechanism of interaction between MSCs and B cells, concerns regarding B cell activation by MSCs in lupus should be diminished.

\section{Acknowledgements}

The authors greatly appreciate Jena Wirth, Medical University of South Carolina, for her critical review of the manuscript. This work was funded by a grant from the Lupus Foundation and NIH grant number UL1 RR029882 (NCRR) and UL1 TR000062 (NCATS) as well as the Medical Research Service, Ralph H. Johnson VAMC.

\section{References}

1. Nauta AJ, Fibbe WE (2007) Immunomodulatory properties of mesenchymal stromal cells. Blood 110: 3499-3506. [Pubmed]

2. D Wang, K Akiyama, H Zhang, T Yamaza, X Li, et al., (2012) Double allogenic mesenchymal stem cells transplantations could not enhance therapeutic effect compared with single transplantation in systemic lupus erythematosus. Clin Dev Immunol.

3. Duffy MM, Ritter T, Ceredig R, Griffin MD (2011) Mesenchymal stem cell effects on T-cell effector pathways. Stem Cell Res Ther 2: 34. [Pubmed] 
Citation: Collins EL, Qi M, Gilkeson G (2015) Effect on B Cell Function by Mesenchymal Stem Cells of Different Derivation. J Stem Cell Res Ther 5: 294. doi:10.4172/2157-7633.1000294

Page 5 of 5

4. Beyth S, Borovsky Z, Mevorach D, Liebergall M, Gazit Z, et al. (2005) Human mesenchymal stem cells alter antigen-presenting cell maturation and induce T-cell unresponsiveness. Blood 105: 2214-2219. [Pubmed]

5. Le Blanc K, Frassoni F, Ball L, Locatelli F, Roelofs H, et al. (2008) Mesenchymal stem cells for treatment of steroid-resistant, severe, acute graft-versus-host disease: a phase II study. Lancet 371: 1579-1586. [Pubmed]

6. Krampera M, Cosmi L, Angeli R, Pasini A, Liotta F, et al. (2006) Role for interferon-gamma in the immunomodulatory activity of human bone marrow mesenchymal stem cells. Stem Cells 24: 386-398. [Pubmed]

7. K Le Blanc, I Rasmusson, B Sundberg, C Götherstrom, M Hassan, et al., (2004) Treatment of severe acute graft-versus-host disease with third party haploidentical mesenchymal stem cells, Lancet. 363: 1439-1441.

8. Corcione A, Benvenuto F, Ferretti E, Giunti D, Cappiello V, et al. (2006) Human mesenchymal stem cells modulate B-cell functions. Blood 107: 367-372. [Pubmed]

9. Che N, Li X, Zhou S, Liu R, Shi D, et al. (2012) Umbilical cord mesenchyma stem cells suppress B-cell proliferation and differentiation. Cell Immunol 274 46-53. [Pubmed]

10. E Traggiai, S Volpi, F Schena, M Gattorno, F Ferlito, et al., (2013) Bone marrow-derived mesenchymal stem cells induce both polyclonal expansion and differentiation of B cells isolated from healthy donors and systemic lupus erythematosus patients. Stem Cells 26: 562-569.

11. Collins E, Gu F, Qi M, Molano I, Ruiz P, et al. (2014) Differential efficacy of human mesenchymal stem cells based on source of origin. J Immunol 193: 4381-4390. [Pubmed]

12. L Sun, D Wang, J Liang, H Zhang, X Feng, et al., (2010) Umbilical cord mesenchymal stem cell transplantation in severe and refractory systemic lupus erythematosus, Arthritis Rheum 62: 2467-2475.

13. Sotiropoulou PA, Perez SA, Gritzapis AD, Baxevanis CN, Papamichail $M$ (2006) Interactions between human mesenchymal stem cells and natural killer cells. Stem Cells 24: 74-85. [Pubmed]

14. M Dominici, K Le Blanc, I Mueller, I Slaper-Cortenbach, F Marini, et al., (2006) Minimal criteria for defining multipotent mesenchymal stromal cells. The International Society for Cellular Therapy position statement, Cytotherapy 8 : 315-317.
15. Pittenger MF, Mackay AM, Beck SC, Jaiswal RK, Douglas R, et al. (1999) Multilineage potential of adult human mesenchymal stem cells. Science 284 143-147. [Pubmed]

16. Yang J, Chu Y, Yang X, Gao D, Zhu L, et al. (2009) Th17 and natural Treg cell population dynamics in systemic lupus erythematosus. Arthritis Rheum 60: 1472-1483. [Pubmed]

17. Menke J, Rabacal WA, Byrne KT, Iwata Y, Schwartz MM, et al. (2009) Circulating CSF-1 promotes monocyte and macrophage phenotypes that enhance lupus nephritis. J Am Soc Nephrol 20: 2581-2592. [Pubmed]

18. Gu Z, Akiyama K, Ma X, Zhang H, Feng X, et al. (2010) Transplantation of umbilical cord mesenchymal stem cells alleviates lupus nephritis in MRL/lpr mice. Lupus 19: 1502-1514. [Pubmed]

19. Chang JW, Hung SP, Wu HH, Wu WM, Yang AH, et al. (2011) Therapeutic effects of umbilical cord blood-derived mesenchymal stem cell transplantation in experimental lupus nephritis. Cell Transplant 20: 245-257. [Pubmed]

20. E.W. Choi, I.S. Shin, S.Y. Park, J.H. Park, J.S. Kim, E.J. Yoon, et al., (2012) Reversal of serologic, immunologic, and histologic dysfunction in mice with systemic lupus erythematosus by long-term serial adipose tissue-derived mesenchymal stem cell transplantation, Arthritis Rheum 64: 243-253.

21. Dörner T, Giesecke C, Lipsky PE (2011) Mechanisms of B cell autoimmunity in SLE. Arthritis Res Ther 13: 243. [Pubmed]

22. Che N, Li X, Zhang L, Liu R, Chen H1, et al. (2014) Impaired B cell inhibition by lupus bone marrow mesenchymal stem cells is caused by reduced CCL2 expression. J Immunol 193: 5306-5314. [Pubmed]

23. Ren G, Zhang L, Zhao X, Xu G, Zhang Y, et al. (2008) Mesenchymal stem cellmediated immunosuppression occurs via concerted action of chemokines and nitric oxide. Cell Stem Cell 2: 141-150. [Pubmed]

24. Collins E, Gilkeson G (2013) Hematopoetic and mesenchymal stem cell transplantation in the treatment of refractory systemic lupus erythematosuswhere are we now? Clin Immunol 148: 328-334. [Pubmed]

25. Rosado MM, Bernardo ME, Scarsella M, Conforti A, Giorda E et al. (2015) Inhibition of B-cell proliferation and antibody production by mesenchymal stromal cells is mediated by T cells. Stem Cells Dev 24: 93-103. [Pubmed] 